\title{
Knapp, klar, übersichtlich
}

\section{Hanspeter Fuhrer}

Dr. med., Facharzt für Allgemeine Innere Medizin, Mitglied FMH

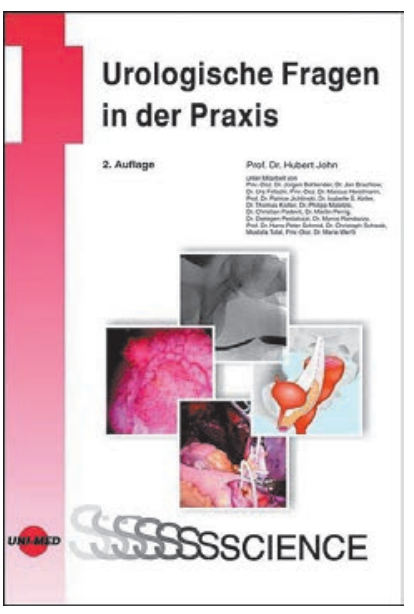

Urologische Fragen in der Praxis

Prof. Dr. Hubert John (Hrsg.)

Reihe UNI-MED Science

2., neubearbeitete Auflage Bremen: UNI-MED Verlag; 2017.

128 Seiten, 73 Abbildungen, Hardcover

ISBN 978-3-8374-1554-4

In der hausärztlichen Praxis stellen sich häufig urologische Fragen zur Abklärung und Therapie von Patientinnen und Patienten. Das von Spezialisten geschriebene "Handbuch" zu urologischen Fragen scheint mir für uns "Grundversorgerinnen und -versorger» sehr

\section{Gute Zusammenfassungen schliessen einzelne} wesentliche Kapitel und Unterkapitel ab.

hilfreich. Knapp, klar und übersichtlich geschrieben und mit einem guten Inhaltsverzeichnis - das ich allerdings eher nach der Bedeutung urologischer Probleme in der Praxis gliedern würde - sowie einem detaillierten Index versehen, trägt es zur Klärung unserer Fragen bei. Gute Zusammenfassungen schliessen einzelne wesentliche Kapitel und Unterkapitel ab.

\section{Reizthema «PSA-Screening»}

Ein viel diskutiertes Problem in der hausärztlichen Praxis ist die Bestimmung des PSA-Wertes, seit er als
Screening-Parameter in Frage gestellt und damit für viele Ärztinnen und Ärzte obsolet wurde. Wann soll er bestimmt werden? Wie soll der Patient über seine Bedeutung informiert werden?

Im interessanten und für Hausärztinnen und -ärzte hilfreichen 4. Kapitel "PSA und Prostatakarzinom» wird die Patienteninformation über die möglichen Konsequenzen eines PSA-Tests als Voraussetzung für eine Abklärung erläutert. Epidemiologische Daten und

Die verschiedenen Therapieoptionen bei einem diagnostizierten Prostatakarzinom werden ausführlich und kritisch dargelegt.

eine differenzierte Interpretation der PSA-Werte führen schliesslich zu einer sinnvollen Anwendung des Tests mit anschliessender Weiterabklärung bei einem positiven Resultat. Die verschiedenen Therapieoptionen bei einem diagnostizierten Prostatakarzinom werden ausführlich und kritisch dargelegt.

Mit dem PSA-Test respektive dem Prostatakarzinom verbunden ist die benigne Prostatahyperplasie. Im 2. Kapitel «Benigne Prostatahyperplasie» wird das weit verbreitete Leiden ausführlich behandelt. Eine wesentliche Neuerung der letzten Jahre ist die primäre, bei einigen Männern gar lebenslange medikamentöse Therapie. Auch Harnverhalt und Katheterismus werden in diesem Zusammenhang besprochen. Im anschliessenden 3. Kapitel «Phytopharmaka bei benigner Prostatahyperplasie» werden Studien vorgestellt, die die Wirksamkeit der Phytotherapie bei Prostatahyperplasie belegen. Phytotherapeutika sind bei dieser Indikation in erster Linie "eine gute initiale Option».

\section{Management der Urolithiasis}

Die Befunde Makrohämaturie, Harnsteinleiden und die Metaphylaxe der Nierensteine werden in den Kapiteln 5 bis 7 dargelegt. Bei der Makrohämaturie wird zwischen schmerzhafter (Urolithiasis, Entzündungen, Traumata und anderen) und schmerzloser (maligne Tumoren) differenziert. Eingehender werden die Problematik der Urolithiasis und die Steinmetaphylaxe, in jeweils einzelne Kapitel gegliedert, ausgeführt. Weitere Ursachen einer Hämaturie sind anschliessend noch 
kurz erwähnt (Medikamente, Endometriose, Zysten und Fehlbildungen).

Das 9. Kapitel, «Harnwegsinfektionen», beginnt mit einem bis zu den alten Griechen zurückreichenden historischen Exkurs über unseren Umgang mit Harnwegsinfektion. Es folgen Definitionen, Epidemiologie, Ätiologie mit Pathogenese und schliesslich eine ausführliche Besprechung von Diagnosen und Therapien der einzelnen Infektionen. Risikofaktoren, Erreger und die Einteilung der chronischen Prostatitis werden in übersichtlicher Form tabellarisch dargestellt. Die verschiedenen Beschwerdebilder, die unter dem Begriff Blasenfunktionsstörungen subsumiert werden, sind in Kapitel 10 dargestellt. Im Abschnitt Therapie werden die verschiedenen mechanischen, medikamentösen und operativen Möglichkeiten besprochen. Therapeutische Optionen sind in diesem Kapitel auch in tabellarischer Form aufgelistet.

Ein eigenes Kapitel (12) ist den Themen «Impotenz und Infertilität» gewidmet. Im ersten Teil werden die verschiedenen Formen der Impotenz gegeneinander abgegrenzt. Die Anamnese ist in eine Sexual- und eine Allgemeinanamnese unterteilt. Hier vermisse ich die wichtige Psychosozialanamnese, da sich die erektile
Dysfunktion sehr oft in diesem "Raum» entwickelt und etabliert. Die notwendigen und möglichen Abklärungen sind in ihrer Vielfalt gut zusammengestellt. Neben den medizinisch-technischen Therapien scheint mir eine psychotherapeutisch orientierte Sexualtherapie wichtig zu sein.

Das praxisorientierte Handbuch ist sehr empfehlenswert, speziell zur Auseinandersetzung mit der Thematik der Prostata.

Weitere Kapitel, auf die hier nicht näher eingegangen wird, betreffen die endokrine Urologie, die Hoden, Nebenhoden und das Skrotum.

\section{In der Gesamtbilanz empfehlenswert}

Zusammenfassend ist das praxisorientierte Handbuch sehr empfehlenswert, speziell zur Auseinandersetzung mit der Thematik der Prostata. Auch die Kapitel «Blasenfunktionsstörungen» und die «Impotenz» beantworten im medizinischen Alltag auftretende Fragen gut und anregend. Gelegentlich vermisste ich einen ganzheitlichen Ansatz im praktischen Umgang mit den leidenden Menschen. 